\title{
DESARROLLO DE LOS ÍNDICES DE SUSCEPTIBILIDAD ELECTROFÍLICA Y NUCLEOFÍLICA
}

\author{
Said Figueredo López ${ }^{* a}$, Manuel Páez Meza ${ }^{a}$, Francisco Torres Hoyos ${ }^{b}$
}

\begin{abstract}
RESUMEN
Dos nuevos índices de reactividad fueron desarrollados dentro del marco conceptual de la Teoría del Funcional de la Densidad. Estos nuevos descriptores, denominados índice de susceptibilidad electrofílica e índice de susceptibilidad nucleofílica, fueron probados para el reproducir las tendencias de reactividad química de series de quinonas halogenadas e indoles 5-sustituidos, respectivamente. Los sistemas moleculares mencionados fueron tratados computacionalmente utilizando el nivel de teoría B3LYP/6-31G(d). Para estos sistemas, los descriptores propuestos aquí mostraron ser más apropiados que otros descriptores ya conocidos, tales como el poder electro-donador, poder electro-aceptador y el comúnmente utilizado índice de electrofilicidad.
\end{abstract}

Palabras clave: reactividad química, índice de susceptibilidad electrofílica, índice de susceptibilidad nucleofílica, comput conceptual.

\section{DEVELOPMENT OF THE ELECTROPHILIC AND NUCLEOPHILIC SUSCEPTIBILITY INDICES}

\begin{abstract}
Two new reactivity indices were developed within the conceptual framework of the Density Functional Theory. These new descriptors, called electrophilic susceptibility index and nucleophilic susceptibility index, were tested to reproduce the chemical reactivity trends of series of halogen-substituted quinones and 5-substituted indoles, respectively. The above mentioned molecular system were computationally treated by using the B3LYP/6-31G(d) level of theory. For these systems, the proposed descriptors showed to be more appropriate than other descriptors currently known such as the electrodonating power, the electroaccepting power and the popular electrophilicity index.
\end{abstract}

Key words: chemical reactivity, electrophilic susceptibility index, nucleophilic susceptibility index, conceptual DFT.

\footnotetext{
a. Facultad de Ciencias Básicas, Fisicoquímica de Mezclas Líquidas, Universidad de Córdoba, Cra. 6 No 74-103, Montería, Colombia, saifer87@gmail.com

${ }^{\mathrm{b}}$ Facultad de Ciencias Básicas, Materiales y Física Aplicada, Universidad de Córdoba, Cra. 6 Nº 74-103, Montería, Colombia
} 


\section{INTRODUCCIÓN}

La Teoría del Funcional de la Densidad (DFT, por sus siglas en inglés) ${ }^{1,2}$ es un formalismo mecano-cuántico en el que la variable principal es la densidad de probabilidad electrónica, $\rho(x$, $y, z$ ) a partir de la cual es posible, en principio, determinar todas las propiedades de sistemas electrónicos, tales como átomos, iones y moléculas. ${ }^{2}$ Desde finales de los años 90 , esta teoría ha mostrado un crecimiento notable en el campo de los cálculos de estructura electrónica de moléculas y en física del estado sólido, lo que ha sido impulsado por la precisión de los cálculos cuantitativos y por su bajo costo computacional. ${ }^{3}$ En particular, un enfoque paralelo de la DFT conllevó al desarrollo de descriptores que proporcionan ideas cualitativas y que permiten la cuantificación de conceptos químicos que solo habían sido tratados de forma intuitiva. ${ }^{3}$ Este campo de investigación, conocido comúnmente como DFT conceptual, ${ }^{1,3}$ surgió como resultado de diversas investigaciones en la University of North Carolina en Chapell Hill, bajo el liderazgo de Robert G. Parr desde los años 80. Concretamente, la DFT conceptual ha provisto diversas representaciones, donde las respuestas de los sistemas electrónicos frente a los cambios en el número de electrones $N$, el potencial externo $v(r)$ y/o la densidad electrónica $\rho(r)$, se han identificado como funciones a partir de las cuales es posible caracterizar diversas propiedades relacionadas con la reactividad química. ${ }^{3}$ Por ejemplo, la representación canónica expresa las propiedades del sistema en términos de $N$ y $v(r),{ }^{1}$ de este modo, cuando un agente externo interactúa con el sistema, el cambio en la energía asociado a las perturbaciones de $N$ y $v(r)$ puede ser escrito mediante la siguiente expansión de Taylor de segundo orden: ${ }^{3}$

$$
\begin{gathered}
\Delta E \equiv E[N+\Delta N, v(r)+\Delta v(r)]-E[N, v(r)]= \\
\left(\frac{\partial E}{\partial N}\right)_{v(r)} \Delta N+\int\left(\frac{\delta E}{\delta v(r)}\right)_{N} \delta v(r) d(r)+ \\
\frac{1}{2 !}\left\{\left(\frac{\partial^{2} E}{\partial^{2} N}\right)_{v(r)}(\Delta N)^{2}+2 \int\left(\frac{\partial}{\partial N}\left(\frac{\delta E}{\delta v(r)}\right)_{N}\right)_{v(r)} \Delta N \delta v(r) d(r)+\iint\left(\frac{\delta^{2} E}{\delta v^{2}(r)}\right)_{N} \delta v(r) \delta v\left(r^{\prime}\right) d r d r^{\prime}\right\}
\end{gathered}
$$

Dentro de este esquema se ha considerado que uno de los principales objetivos de la DFT conceptual ha sido darle significado físico a las derivadas de la energía que aparecen en la ecuación $1 .{ }^{3}$ Concretamente, la primera derivada de la energía, con respecto al número de electrones, es el potencial químico electrónico $\mu,{ }^{1,3}$ una propiedad que ha sido identificada como la tendencia de los electrones a escapar desde un sistema en equilibrio: ${ }^{4}$

$$
\mu=\left(\frac{\partial E}{\partial N}\right)_{v(r)}
$$


La dureza $\eta$, que aparece en la ecuación 1 como la segunda derivada de la energía con respecto al número de electrones, ha sido reconocida como una propiedad que mide la resistencia de los electrones a ser transferidos desde un sistema molecular en equilibrio: ${ }^{3,4}$

$$
\eta=\left(\frac{\partial^{2} E}{\partial N^{2}}\right)_{v(r)}
$$

En 1999, Parr, Szentpaly y Liu, ${ }^{5}$ demostraron que cuando un sistema es saturado con electrones provenientes de los alrededores manteniendo el potencial externo constante, ocurre una disminución en la energía que es equivalente a $-\mu 2 / 2 \eta$. El valor absoluto de esta cantidad fue definido como el índice de electrofilicidad:

$$
\omega=\frac{\mu^{2}}{2 \eta}
$$

Por lo tanto, valores altos de $\omega$ serán característicos para los sistemas moleculares más propensos a interactuar covalentemente con especies nucleofílicas. No obstante, aunque el índice de electrofilicidad ha sido utilizado eficazmente para el estudio de la reactividad de diversos sistemas moleculares, ${ }^{4,6}$ su descripción de la reactividad relativa ha resultado ser inconclusa en algunos casos. ${ }^{7}$ Similarmente, los índices de filicidad propuestos por Chattaraj, ${ }^{8}$ y que se derivan del concepto de electrofilicidad, no dan información adicional a la proporcionada por la suavidad local ${ }^{3}$ y las funciones de Fukui ${ }^{3,9}$ cuando se estudian aspectos relacionados con la reactividad intramolecular, además, para tendencias de reactividad intermolecular, los índices de filicidad pueden ser utilizados solo en limitados casos. ${ }^{9}$ Con la intención de obtener descriptores de reactividad más consistentes con las observaciones experimentales, diversos trabajos han sido realizados en el ámbito de la DFT conceptual,, 10 incluyendo la publicación concerniente a los índices definidos como poder electroaceptador y poder electrodonador, ${ }^{9,10}$ además del trabajo recientemente publicado por Morell y colaboradore ${ }^{10}$ donde se introdujeron nuevos índices locales de electrofilicidad y nucleofilicidad. Con el ánimo de contribuir a esta serie de avances en el campo de la DFT conceptual, en el presente trabajo se propone el desarrollo de dos nuevos índices globales de reactividad, denominados índice de susceptibilidad electrofílica e índice de susceptibilidad nucleofílica, respectivamente. Para validar el uso de estos índices de reactividad para sistemas moleculares, se compararon los resultados teóricos de reactividad de series de indoles y derivados quinona halo-sustituidos con los respectivos parámetros experimentales de reactividad. Los resultados obtenidos muestran que los índices de reactividad propuestos describen apropiadamente la reactividad química de los sistemas moleculares considerados.

\section{PARTE EXPERIMENTAL}

Con el propósito de validar la utilización de los índices de susceptibilidad nucleofílica $\left(\Delta \theta^{-}\right)$ y electrofílica $\left(\Delta \theta^{+}\right)$, estos fueron calculados respectivamente para series de indoles (figura 1) y halo-quinonas (figura 2), cuyos parámetros de reactividad son experimentalmente 
conocidos. Además, los valores de $\Delta \theta^{-}$fueron comparados con los del recíproco del poder electro-donador $\left(1 / \omega^{-}\right) ;^{9}$ mientras que la predictividad de $\Delta \theta^{-}$fue comparada con la del índice de electrofilicidad $(\omega)^{5}$ y del poder electro-aceptador $\left(\omega^{+}\right) .{ }^{9}$ Computacionalmente, todas las geometrías moleculares fueron diseñadas y pre-optimizadas por mecánica molecular mediante el Campo de Fuerzas Universal (UFF, por sus siglas en inglés) implementado en el programa Avogadro; ${ }^{11}$ posteriormente, las geometrías de equilibrio fueron optimizadas analíticamente utilizando el programa NWChem $6.3^{12}$ al nivel de teoría B3LYP/6-31G(d), como fue propuesto por Domingo y colaboradores ${ }^{13}$ para el tratamiento teórico de los mismos indoles estudiados aquí.

\section{RESULTADOS Y DISCUSIÓN}

\section{Desarrollo del índice de susceptibilidad electrofílica}

Manteniendo el potencial externo constante, el cambio instantáneo de la energía asociado a la respuesta de un sistema (modelado por el ensamble canónico) a la aceptación de carga eléctrica fue definido por la siguiente expansión de Taylor de segundo orden:

$$
\Delta E^{+}=-\Delta \mu^{+} \Delta N^{+}+\frac{1}{2} \eta^{+}\left(\Delta N^{+}\right)^{2}
$$

Donde el potencial químico inicial es $\mu_{0}^{+}$, aumentando su valor hasta cero cuando el sistema es saturado con electrones. Expresando el potencial químico mediante una expansión de Taylor alrededor del número de electrones del sistema de referencia se tiene: ${ }^{3}$

$$
\begin{gathered}
\mu^{+}=\mu_{0}^{+}+\left(\frac{\partial \mu^{+}}{\partial N^{+}}\right)_{v(r)} \Delta N^{+} \\
\mu^{+}=\mu_{0}^{+}+\eta^{+} \Delta N^{+}
\end{gathered}
$$

Por lo que $\Delta \mu^{+}$se puede aproximar como:

$$
\Delta \mu^{+}=\eta^{+} \Delta N^{+}
$$

Introduciendo este resultado en la ecuación 5, se obtiene la siguiente expresión para el cambio en la energía del sistema:

$$
\Delta E^{+}=-\frac{1}{2} \eta^{+}\left(\Delta N^{+}\right)^{2}
$$

Considerando ahora que el cambio en el número de electrones permanece proporcional a $-\mu^{+}{ }_{0}$ $/ \eta^{+}$, es posible $\Delta N^{+}$definir a por la expresión:

$$
\Delta N^{+}=-\frac{\mu_{0}^{+}}{\eta^{+}} \cdot g^{+}
$$


Aquí, $g^{+}$es una función que describe la evolución del número de electrones durante el proceso de aceptación de carga, y cuyo valor inicial es 0 tendiendo a 1 cuando el sistema es saturado con electrones, es decir, cuando $\mu^{+}$se aproxima a 0 . La derivada de $g^{+}$con respecto al número de electrones se obtiene derivando implícitamente la ecuación 10:

$$
\left(\frac{\partial g^{+}}{\partial N}\right)_{v(r)}=\frac{g^{+} \cdot \gamma^{+}}{\eta^{+}}-\frac{\eta^{+}}{\mu_{0}^{+}}
$$

Donde $\gamma$ es la derivada de la dureza con respecto a $N$ (hiperdureza): ${ }^{14}$

$$
\gamma=\left(\frac{\partial \eta}{\partial N}\right)_{v(r)}
$$

A partir de la ecuación 10, la ecuación 9 se re-escribe como:

$$
\Delta E^{+}=-\frac{1}{2} \frac{\left(\mu_{0}^{+}\right)^{2}}{\eta^{+}} \cdot\left(g^{+}\right)^{2}
$$

De esta manera, se define la función de susceptibilidad electrofílica $\theta^{+}$como:

$$
\theta^{+}=\frac{1}{2} \frac{\left(\mu_{0}^{+}\right)^{2}}{\eta^{+}} \cdot\left(g^{+}\right)^{2}
$$

La máxima estabilización del sistema ocurre cuando $g^{+}=1$, en cuyo caso $\theta^{+}$se convierte en el concepto de poder electro-aceptador $\left(\omega^{+}\right){ }^{9,10}$

$$
\omega^{+}=\lim _{g^{+} \rightarrow 1} \theta^{+}=\frac{1}{2} \frac{\left(\mu_{0}^{+}\right)^{2}}{\eta^{+}}
$$

Por otro lado, la derivada de la función de susceptibilidad electrofílica con respecto a $N$ está dada por:

$$
\begin{gathered}
\left(\frac{\partial \theta^{+}}{\partial N}\right)_{v(r)}=\frac{\left(\mu_{0}^{+}\right)^{2}}{2}\left[-\frac{\gamma^{+}\left(g^{+}\right)^{2}}{\left(\eta^{+}\right)^{2}}+\frac{2 g}{\eta^{+}}\left(\frac{\partial g^{+}}{\partial N}\right)_{v(r)}\right] \\
\left(\frac{\partial \theta^{+}}{\partial N}\right)_{v(r)}=\frac{\left(\mu_{0}^{+}\right)^{2}}{2}\left[\frac{\gamma^{+}\left(g^{+}\right)^{2}}{\left(\eta^{+}\right)^{2}}-\frac{2 g^{+}}{\mu_{0}^{+}}\right]
\end{gathered}
$$

En la situación límite en la que el sistema de referencia es saturado con la máxima cantidad de electrones, el valor de $g^{+}$tiende a 1 , mientras que $\Delta N^{+}$alcanza su máximo valor: $\Delta N^{+}{ }_{\max }=-\mu^{+}{ }_{0}$ $\eta^{+}$. En estas condiciones, se define el índice de susceptibilidad electrofílica como:

$$
\Delta \theta^{+}=\lim _{g^{+} \rightarrow 1}\left(\frac{\partial \theta^{+}}{\partial N}\right)_{v(r)}=\frac{1}{2} \frac{\left(\mu_{0}^{+}\right)^{2} \gamma^{+}}{\left(\eta^{+}\right)^{2}}-\mu_{0}^{+}
$$


Ya que el potencial químico es siempre negativo, se espera que valores altos positivos de $\Delta \theta^{+}$sean característicos de sistemas moleculares altamente electrofílicos.

\section{Desarrollo del índice de susceptibilidad nucleofílica}

Manteniendo el potencial externo constante, el cambio instantáneo del gran potencial asociado a la respuesta de un sistema (modelado por el ensamble gran canónico) a la donación de carga eléctrica está dado por la siguiente expansión de Taylor de segundo orden: ${ }^{10}$

$$
\Delta \Omega^{-}=-\Delta \mu^{-} \Delta N^{-}-\frac{1}{2} S^{-}\left(\Delta \mu^{-}\right)^{2}
$$

Donde el potencial químico inicial es $\mu_{0}^{-}$, aumentando su valor hasta cero cuando el sistema dona la máxima cantidad de electrones. Ya que $\Delta N^{-}$es negativo, $\Delta \mu^{-}$debe expresarse como:

$$
\Delta \mu^{-}=-\eta^{-} \Delta N^{-}
$$

Introduciendo este resultado en la ecuación 18, se obtiene la siguiente expresión para el cambio en el gran potencial del sistema:

$$
\Delta \Omega^{-}=\frac{1}{2} \eta^{-}\left(\Delta N^{-}\right)^{2}
$$

Asumiendo que el cambio en el número de electrones permanece proporcional a $\mu^{-} / \eta^{-},{ }^{10}$ es posible definir a $\Delta N^{-}$por la expresión:

$$
\Delta N^{-}=\frac{\mu_{0}^{-}}{\eta^{-}} \cdot g^{-}
$$

Nuevamente, se ha introducido una función $g^{-}$que describe la evolución del número de electrones durante el proceso de donación de carga, y cuyo valor inicial es 0 tendiendo a 1 cuando el sistema dona la máxima cantidad de electrones, es decir, cuando $\mu^{-}$se aproxima a 0 . La derivada de $g$ con respecto al número de electrones se obtiene derivando implícitamente la ecuación 21:

$$
\left(\frac{\partial g^{-}}{\partial N}\right)_{v(r)}=\frac{\eta^{-}}{\mu_{0}^{-}}-\frac{g^{-} \cdot \gamma^{-}}{\eta^{-}}
$$

Utilizando el resultado de la ecuación 21, es posible re-escribir la ecuación 20 como:

$$
\Delta \Omega^{-}=\frac{1}{2} \frac{\left(\mu_{0}^{-}\right)^{2}}{\eta^{-}} \cdot\left(g^{-}\right)^{2}
$$

Ahora, se define la función de susceptibilidad nucleofílica $\theta^{-}$como el recíproco de $\Delta \Omega^{-}$:

$$
\theta^{-}=\frac{2 \eta^{-}}{\left(\mu_{0}^{-}\right)^{2}} \cdot \frac{1}{\left(g^{-}\right)^{2}}
$$


La máxima desestabilización del sistema ocurre cuando $g^{-}=1$, en cuyo caso $\theta^{-}$se convierte en el recíproco del concepto de poder electro-donador $\left(\omega^{-}\right):{ }^{9,10}$

$$
\frac{1}{\omega^{-}}=\lim _{g^{-} \rightarrow 1} \theta^{-}=\frac{2 \eta^{-}}{\left(\mu_{0}^{-}\right)^{2}}
$$

Por otro lado, la derivada de la función de susceptibilidad nucleofílica con respecto a $N$ está dada por:

$$
\begin{gathered}
\left(\frac{\partial \theta^{-}}{\partial N}\right)_{v(r)}=\frac{2}{\left(\mu_{0}^{-}\right)^{2}}\left[\frac{\gamma^{-}}{\left(g^{-}\right)^{2}}-\frac{2 \eta^{-}}{\left(g^{-}\right)^{3}}\left(\frac{\partial g^{-}}{\partial N}\right)_{v(r)}\right] \\
\left(\frac{\partial \theta^{+}}{\partial N}\right)_{v(r)}=\frac{2}{\left(\mu_{0}^{-}\right)^{2}}\left[\frac{3 \gamma^{-}}{\left(g^{-}\right)^{2}}-\frac{2\left(\eta^{-}\right)^{2}}{\mu_{0}^{-}\left(g^{-}\right)^{3}}\right]
\end{gathered}
$$

En la situación límite en la que el sistema de referencia dona la máxima cantidad de electrones, el valor de $g^{-}$tiende a 1 , mientras que $\Delta N^{-}$alcanza su máximo valor: $\Delta N_{\text {max }}^{-}=\mu_{0}^{-} \eta^{-}$. De esta forma, se define el índice de susceptibilidad nucleofílica como:

$$
\Delta \theta^{-}=\lim _{g^{-} \rightarrow 1}\left(\frac{\partial \theta^{-}}{\partial N}\right)_{v(r)}=6 \frac{\gamma^{-}}{\left(\mu_{0}^{-}\right)^{2}}-4 \frac{\left(\eta^{-}\right)^{2}}{\left(\mu_{0}^{-}\right)^{3}}
$$

Finalmente, debido a que el potencial químico es siempre negativo, valores altos positivos de $\Delta \theta^{-}$deberán ser característicos de sistemas moleculares altamente nucleofílicos.

La selección de las moléculas empleadas para la validación de los índices $\Delta \theta^{+}$y $\Delta \theta^{-}$en el presente trabajo se realizó con base en la disponibilidad de información experimental sobre la reactividad química de las mismas; en particular, la reactividad nucleofílica de la serie de indoles 5-sustituidos fue medida frente al 4,6-dinitrobenzofuroxano (DNBF) y reportada por Lakhdar y colaboradores ${ }^{15}$ en términos del parámetro de nucleofilicidad de $\operatorname{Mayr}(N)$; mientras que los datos experimentales de reactividad electrofílica de las halo-quinonas fueron obtenidos por su reacción con siloaxialquenos, enaminas y aminas, y reportados por Guo y Mayr $^{16}$ mediante el parámetro de electrofilicidad E. Los índices de susceptibilidad nucleofílica y el poder electro-donador utilizados para reproducir la reactividad nucleofílica de los indoles, fueron calculados respectivamente por:

$$
\begin{gathered}
\Delta \theta^{-}=6 \frac{\gamma}{\left(\mu^{-}\right)^{2}}-4 \frac{\eta^{2}}{\left(\mu^{-}\right)^{3}} \\
\omega^{-}=\frac{1}{2} \frac{\left(\mu^{-}\right)^{2}}{\eta}
\end{gathered}
$$


donde se han removido los sub-índices 0 , y además se ha utilizado el esquema de interpolación de Gázquez, Cedillo y Vela ${ }^{9}$ para el cálculo de los potenciales químicos y la dureza:

$$
\begin{gathered}
\eta^{+}=\eta^{-}=\eta=\frac{1}{2}(I-A) \\
\mu^{-}=-\frac{1}{4}(3 I+A) \\
\mu^{+}=-\frac{1}{4}(I+3 A)
\end{gathered}
$$

Los valores de la energía de ionización $(I)$ y afinidades electrónicas $(A)$ fueron calculados de acuerdo al esquema propuesto por Vargas y colaboradores. ${ }^{17}$ Por otra parte, los valores de la hiper-dureza fueron aproximados mediante el teorema de Koopmans: ${ }^{18}$

$$
\gamma=\varepsilon_{\text {LUMO }}-2 \varepsilon_{\text {HOMO }}+\varepsilon_{\text {HOMO-1 }}
$$

Por otro lado, los valores de los índices de susceptibilidad electrofílica, poder electro-donador, e índice de electrofilicidad fueron calculados respectivamente como:

$$
\begin{gathered}
\Delta \theta^{+}=\frac{1}{2} \frac{\left(\mu^{+}\right)^{2} \gamma}{\eta^{2}}-\mu^{+} \\
\omega^{+}=\frac{1}{2} \frac{\left(\mu^{+}\right)^{2}}{\eta} \\
\omega=\frac{1}{2} \frac{\mu^{2}}{\eta}
\end{gathered}
$$

donde el potencial químico $\mu$ está dado como el promedio de los valores de $\mu^{+}$y $\mu^{-} .9$ Los valores de los descriptores de reactividad de las moléculas estudiadas fueron registrados en la tabla 1 junto con los respectivos valores experimentales de los parámetros de reactividad. 
Tabla 1. Valores de los descriptores de reactividad de las moléculas estudiadas.

\begin{tabular}{ccccc}
\hline indoles & $\Delta \theta^{-}$ & $1 / \omega^{-}$ & $N$ & \\
1m & 4,738 & 0,8764 & 7,22 & \\
1n & 3,928 & 0,7425 & 6,44 & \\
1c & 4,059 & 0,7647 & 6,22 & \\
1g & 3,882 & 0,7299 & 6,00 & \\
1a & 3,810 & 0,7202 & 5,55 & \\
1f & 3,261 & 0,6394 & 4,42 & \\
1o & 2,847 & 0,5939 & 3,97 & \\
1d & 2,606 & 0,5477 & 2,83 & \\
halo-quinonas & $\Delta \theta^{+}$ & $\omega^{+}$ & $\omega$ & $E$ \\
1 & 3,350 & 0,5068 & 1,607 & $-16,19$ \\
1b & 3,839 & 0,6843 & 1,862 & $-16,11$ \\
1c & 3,983 & 0,8089 & 2,001 & $-13,84$ \\
1d & 4,021 & 0,7396 & 1,981 & $-11,12$ \\
1e & 3,656 & 2,833 & 4,306 & $-12,02$ \\
\hline Valores de $\Delta \theta^{-}$y $1 / \omega^{-}$en $e V^{1}, \Delta \theta^{+}, \omega^{+}$y $\omega$ en $e V$
\end{tabular}

\section{Reactividad nucleofílica de indoles 5-sustituidos}

Con respecto a la reactividad de los indoles $1 \mathrm{~m}$ a $1 \mathrm{~d}$, se observa que los valores de $\Delta \theta^{-}$y $1 / \omega^{-}$ dieron el mismo orden relativo de reactividad, siendo $1 \mathrm{~m}$ y $1 \mathrm{~d}$ los derivados con menor y mayor carácter nucleofílico, respectivamente. De este modo, la escala de reactividad dada por ambos descriptores difiere de la escala experimental de reactividad solo en el orden relativo de los compuestos $1 \mathrm{n}$ y $1 \mathrm{c}$, ya que las medidas experimentales del parámetro $N$ sugieren que 1c es más nucleofílico. Por lo tanto, ya que la descripción cualitativa de reactividad dada por el índice de susceptibilidad es equivalente a la del recíproco del poder electro-donador, se infiere que $\Delta \theta^{-}$es un descriptor de reactividad con potencialidad para el tratamiento teórico de la reactividad nucleofílica de sistemas moleculares. Adicionalmente, los valores de $\Delta \theta^{-}$y $1 / \omega^{-}$ fueron correlacionados con los de $N$ mediante análisis de regresión lineal, donde se tomaron los valores experimentales como variable dependiente. En este contexto, los coeficientes de determinación lineal R2 exhibieron valores de 0,9546 y 0,9462 para $N$ como función de $\Delta \theta^{-}$y $1 / \omega^{-}$, respectivamente, por lo que se infiere que, desde el punto de vista estadístico los valores de $\Delta \theta^{-}$representan más apropiadamente las variaciones experimentales observadas en el parámetro de reactividad $N$. Así, se concluye que en comparación con $1 / \omega^{-}, \Delta \theta^{-}$es un mejor descriptor de nucleofilicidad para el estudio teórico de la reactividad de la serie de indoles considerados aquí. 


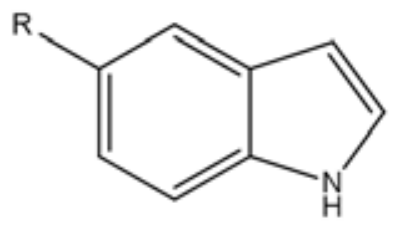
lm. $\mathrm{R}=\mathrm{NH}_{2}$
1a. $\mathrm{R}=\mathrm{H}$
ln. $\mathrm{R}=\mathrm{OH}$
lf. $\mathrm{R}=\mathrm{Cl}$
1c. $\mathrm{R}=\mathrm{OCH}_{3}$
1.. $\mathrm{R}=\mathrm{COOH}$
lg. $\mathrm{R}=\mathrm{CH}_{3}$
1d. $\mathrm{R}=\mathrm{CN}$

Figura 1. Estructuras de los indoles 5-sustituidos estudiados en el presente trabajo.

\section{Reactividad electrofílica de quinonas halogenadas}

Para la serie de quinonas halogenadas la a 1e, tres descriptores electrofílicos fueron utilizados para reproducir los datos experimentales de reactividad, incluyendo el índice de susceptibilidad desarrollado en el presente trabajo, así como los índices de electrofilicidad y el poder electro-donador. Los valores del parámetro de electrofilicidad E de estos compuestos dan el orden de reactividad 1a-1b-1c-1e-1d, siendo 1d el más electrofílico. Desde el punto de vista teórico, los valores de $\omega^{+}$y $\omega$ dieron lugar a una misma escala de reactividad que difiere de la escala experimental en el orden de reactividad de los derivados 1c y 1d, además del hecho de que estos descriptores resultaron sobre-estimar la reactividad de la quinona 1e. Concretamente, aunque se esperaría que $1 \mathrm{~d}$ mostrara un valor más alto que $1 \mathrm{c}$ en cuanto a los descriptores electrofílicos, los valores de $\omega^{+}$y $\omega$ obtenidos para 1c fueron mayores que los de 1d; además los valores de $\omega^{+}$y $\omega$ para la quinona 1e resultaron ser varias veces mayor en magnitud en comparación con los del derivado más electrofílico (1d), lo que obviamente va en contra de lo experimentalmente observado.<smiles>O=C1C=CC(=O)C=C1</smiles><smiles>O=C1C(F)=C(F)C(=O)C(F)=C1F</smiles>

d<smiles>O=C1C=C(Cl)C(=O)C=C1Cl</smiles>

b<smiles>O=C1C(Cl)=C(Cl)C(=O)C(Cl)=C1Cl</smiles>

c<smiles>O=C1C(=O)C(Cl)=C(Cl)C(Cl)=C1Cl</smiles>

Figura 2. Estructuras de las halo-quinonas estudiadas. 
Por otro lado, los valores del índice de susceptibilidad electrofílica $\Delta \theta^{+}$mostraron una correcta descripción de la reactividad relativa de los compuestos 1a a 1d, no obstante, $\Delta \theta^{+}$ sub-estimó la reactividad del derivado quinona 1e por debajo de la reactividad de 1b. Aun así, en comparación con $\omega^{+}$y $\omega$, la reactividad electrofílica dada por $\Delta \theta^{+}$fue más consistente con lo experimentalmente observado para la serie de quinonas halogenadas.

\section{CONCLUSIONES}

Utilizando las representaciones canónica y gran canónica de la DFT conceptual, se modelaron los cambios en la energía y gran potencial, respectivamente, a través de un formalismo paralelo al propuesto en otros trabajos. ${ }^{5,9,10}$ De esta forma, se definieron las funciones de susceptibilidad electrofílica y nucleofílica, que equivalen a los conceptos de poder electro-aceptador y poder electro-donador cuando el sistema de referencia se encuentra en la situación límite en la que es saturado con electrones y cuando dona la máxima cantidad de carga, respectivamente. El índice de susceptibilidad electrofílica $\left(\Delta \theta^{+}\right)$fue introducido formalmente como la razón de cambio de la función de susceptibilidad electrofílica en el límite en el que el sistema es saturado con electrones; por otro lado, se definió el índice de susceptibilidad nucleofílica $\left(\Delta \theta^{-}\right)$como la razón de cambio de la función de susceptibilidad nucleofílica en el límite en el que el sistema dona la máxima cantidad de carga. Empleando el esquema de interpolación bi-parabólico propuesto por Gázquez y colaboradores, ${ }^{9}$ se establecieron las ecuaciones 28 y 34 como las ecuaciones de trabajo para el cálculo de $\Delta \theta^{-}$y $\Delta \theta^{+}$, respectivamente. Con el objetivo de validar el uso de estos nuevos índices de reactividad, estos fueron utilizados para reproducir las tendencias de reactividad de series de indoles 5-sustituidos y quinonas halogenadas. Desde el punto de vista metodológico, la estructura electrónica de los sistemas moleculares mencionados fue tratada utilizando el nivel de teoría B3LYP/6-31G(d), que mostró ser apropiado para los propósitos del trabajo realizado. En general, los resultados obtenidos mostraron que los índices $\Delta \theta^{-}$y $\Delta \theta^{+}$resultaron ser más apropiados que otros descriptores de reactividad tales como el poder electro-donador y los índices de electrofilicidad para el estudio teórico de la reactividad de los indoles y quinonas, respectivamente, por lo tanto, estos nuevos índices podrían ser sometidos a estudios adicionales con el propósito de corroborar su potencialidad para el tratamiento teórico de la reactividad química de sistemas moleculares más complejos.

\section{AGRADECIMIENTO}

Los autores agradecen a la Universidad de Córdoba por el apoyo prestado para la realización del presente trabajo. 


\section{REFERENCIAS BIBLIOGRÁFICAS}

1. Parr RG, Yang W. "Density-functional theory of atoms and molecules". 16va ed. New York: Oxford university press; 1989.

2. Cramer CJ. "Essentials of computational chemistry: theories and models". 2da ed. Chichester: John Wiley \& Sons; 2013.

3. Proft FD, Ayers PW, Geerlings P. The Conceptual Density Functional Theory Perspective of Bonding. En Frenking G, Shaik S. Edit. Chemical Bond: Fundamental aspects of chemical bonding. Weinheim: Wiley-VCH Verlag GmbH \& Co. KGaA; 2014. p 233270.

4. Martínez-Araya JI, Salgado-Morán G, Glossman-Mitnik D. Computational Nutraceutics: Chemical Reactivity Properties of the Flavonoid Naringin by Means of Conceptual DFT. J Chem. 2013; 2013: 850297.

5. Parr RG, Szentpaly L v, Liu S. Electrophilicity index. J Am Chem Soc. 1999; 121(9): 1922-4.

6. Singh O, R Kakularam K, Reddanna P, Aparoy P. Understanding the dual inhibition of COX-2 and Carbonic anhydrase-II by Celecoxib and CG100649 using Density functional theory calculations and other Molecular Modelling approaches. Protein Pept Lett. 2015; 22(10): 903-12.

7. Mendoza Huizar LH, Rios-Reyes CH, Olvera-Maturano NJ, Robles J, Rodriguez JA. Chemical reactivity of quinclorac employing the HSAB local principle-Fukui function. Open Chem. 2015; 13(1): 52-60.

8. Chattaraj PK, Maiti B, Sarkar U. Philicity: a unified treatment of chemical reactivity and selectivity. J Phys Chem A. 2003; 107(25): 4973-5.

9. Orozco-Valencia ÁU, Vela A. The Electrodonating and Electroaccepting Powers in Atoms. J Mex Chem Soc. 2012; 56(3):294-301.

10. Morell C, Gázquez JL, Vela A, Guégan F, Chermette H. Revisiting electroaccepting and electrodonating powers: proposals for local electrophilicity and local nucleophilicity descriptors. Phys Chem Chem Phys. 2014; 16(48): 26832-42.

11. Hanwell MD, Curtis DE, Lonie DC, Vandermeersch T, Zurek E, Hutchison GR. Avogadro: An advanced semantic chemical editor, visualization, and analysis platform. J Cheminformatics. 2012; 4(1):17.

12. Valiev M, Bylaska EJ, Govind N, Kowalski K, Straatsma TP, Van Dam HJ, et al. NWChem: a comprehensive and scalable open-source solution for large scale molecular simulations. Comput Phys Commun. 2010; 181(9): 1477-89.

13. Domingo LR, Pérez $\mathrm{P}$. The nucleophilicity $\mathrm{N}$ index in organic chemistry. Org Biomol Chem. 2011; 9(20): 7168-75.

14. Fuentealba P, Parr RG. Higher-order derivatives in density-functional theory, especially the hardness derivative $\partial \eta / \partial$ N. J Chem Phys. 1991; 94(8): 5559-64.

15. Lakhdar S, Westermaier M, Terrier F, Goumont R, Boubaker T, Ofial AR, et al. Nucleophilic reactivities of indoles. J Org Chem. 2006; 71(24): 9088-95.

16. Guo X, Mayr H. Quantification of the Ambident Electrophilicities of HalogenSubstituted Quinones. J Am Chem Soc. 2014; 136(32): 11499-512.

17. Vargas R, Garza J, Cedillo A. Koopmans-like approximation in the Kohn-Sham method 
and the impact of the frozen core approximation on the computation of the reactivity parameters of the density functional theory. J Phys Chem A. 2005; 109(39): 8880-92.

18. Cárdenas C, Rabi N, Ayers PW, Morell C, Jaramillo P, Fuentealba P. Chemical reactivity descriptors for ambiphilic reagents: dual descriptor, local hypersoftness, and electrostatic potential. J Phys Chem A. 2009; 113(30): 8660-7.

19. Cárdenas C, Rabi N, Ayers PW, Morell C, Jaramillo P, Fuentealba P. Chemical reactivity descriptors for ambiphilic reagents: dual descriptor, local hypersoftness, and electrostatic potential. J. Phys. Chem. A 2009; 113(30): 8660-7. 